\title{
Condom effectiveness against non-viral sexually transmitted infections: a prospective study using electronic daily diaries
}

\author{
Richard A Crosby, ${ }^{1}$ Richard A Charnigo, ${ }^{1}$ Chandra Weathers, ${ }^{1}$ Angela M Caliendo, ${ }^{2}$ \\ Lydia A Shrier ${ }^{3}$
}

${ }^{1}$ Department of Health Behavior, College of Public Health at the University of Kentucky, Lexington, Kentucky, USA

${ }^{2}$ Department of Pathology and Laboratory Medicine (Infectious Diseases), Emory University School of Medicine, Atlanta, Georgia, USA

${ }^{3}$ Division of Adolescent/Young Adult Medicine, Children's Hospital Boston and Department of Pediatrics, Harvard Medical School, Boston, Massachusetts, USA

\section{Correspondence to}

Dr Richard A Crosby,

Department of Health Behavior College of Public Health at the University of Kentucky, 151 Washington Avenue, Lexington, KY 40506-0003, USA; crosby@uky.edu

Accepted 8 August 2012 Published Online First 21 September 2012

\begin{abstract}
Objectives To prospectively evaluate the protective value of consistent and correct use of latex condoms against the acquisition of Chlamydia trachomatis, Neisseria gonorrhoeae and Trichomonas vaginalis.

Methods Patients $(\mathrm{N}=929)$ attending clinics that treat sexually transmitted infections (STIs) were prospectively followed for up to 6 months. Urine STI nucleic acid amplification testing was performed at baseline, 3 months and 6 months. Participants were instructed to respond to daily prompts from a handheld device by completing a report for each penile-vaginal sexual intercourse event. Generalised estimating equation models examined associations of consistent as well as consistent and correct condom use with STI incidence over 3-month intervals.
\end{abstract}

Results Consistent condom use was not significantly associated with STI incidence (Estimated OR (EOR) $=0.75 ; 95 \% \mathrm{Cl}$ (Cl) 0.43 to 1.30; $p=0.31$ ). However, individuals who used condoms both correctly and consistently were estimated to have $59 \%$ lower odds of acquiring an STI (EOR $=0.41 ; 95 \% \mathrm{Cl} 0.19$ to 0.90; $\mathrm{p}=.026$ ), compared to those who did not.

Conclusions The correct as well as the consistent use of condoms greatly reduces the odds of non-viral STI acquisition.

\section{INTRODUCTION}

More than a decade ago, the USA Department of Health and Human Services issued a report about the effectiveness of latex condoms for the prevention of sexually transmitted infections (STIs). ${ }^{1}$ Beyond HIV transmission and female-to-male transmission of gonorrhoea, the report concluded evidence was insufficient to judge the protective value of condoms against other STIs. Since the report was issued, findings from a few welldesigned studies suggest a protective value of condoms against male-to-female transmission of genital herpes, ${ }^{2}$ chlamydia/gonorrhoea ${ }^{3}$ syphilis, ${ }^{4}$ and human papillomavirus. ${ }^{5}$ However, most studies conducted in the past 10 years have failed to show a significant association between condom use and STI acquisition. ${ }^{6}$ The majority of these studies have been designed with several inherent forms of bias toward the null hypothesis (ie, condoms are not protective). For example, a misclassification bias is created when a study does not measure and adjust for the incorrect use of condoms (failure to use the device from start to finish of penetrative sex) or for the events of breakage or slippage. ${ }^{6-8}$ In essence, failure to control for condom breakage and slippage may produce the analytical equivalent of condom non-use, which confounds study findings. For example, a prospective study of clinic attendees found 13\% incidence of chlamydia and gonorrhoea among people reporting consistent condom use but also reporting at least one problem with correct use. ${ }^{9}$ In contrast, among those reporting consistent use and a lack of problems (slippage, breakage, leaking, early removal, late application), no incident infections were found.

Past studies have also relied on the validity of retrospective recall, sometimes over periods of time measured in months rather than days or weeks. ${ }^{67}$ Fortunately, the science of collecting valid selfreported data on condom use behaviours has greatly improved in recent years. ${ }^{10}$ In particular, mobile electronic devices enable daily reporting, which may dramatically reduce recall bias and minimise social desirability bias. ${ }^{11}$ In parallel, improved technology has enhanced validity of nucleic acid amplification testing for STI assessment. Also, single-dose therapy to treat prevalent cases provides assurance that subsequent infections are truly incident cases.

Evidence suggests that people may use condoms as a consequence of a correct position that they are likely to have an STI. ${ }^{12}$ This post-infection condom use confounds data analyses by deflating the expected negative association between use and STI acquisition. ${ }^{13}$ The issue is overcome by a prospective design that establishes an infection-free cohort initially and by measuring behaviours over relatively short time intervals. In condom effectiveness studies, the confound of post-infection condom use is eliminated from data analyses based on subjects' consistency $(100 \%$ use) or lack thereof, instead of their relative frequencies of condom use. For example, someone who uses condoms $40 \%$ of the time, becomes infected, and then starts using condoms $80 \%$ of the time because of the infection is (appropriately) not deemed a superior condom user in the analytic approach of comparing 100\% users to the remainder.

The purpose of this study was to prospectively determine the protective value of consistent and correct condom use against urethral/vaginal acquisition of three common STIs (Chlamydia trachomatis, Neisseria gonorrhoeae and Trichomonas 
vaginalis) using daily electronic assessments and nucleic acid amplification test technology. First, we tested the hypothesis that consistent condom use would have a significant protective effect against the three STIs. Subsequently, we tested whether consistent and correct condom use would have a protective effect.

\section{METHODS \\ Study design}

Outpatients were recruited from five clinics caring for individuals at high-risk for STI in three US cities: a publicly-funded STI clinic in Southern USA; a publicly-funded STI clinic in midwestern USA; and an STI clinic of a large teaching hospital and two adolescent medicine clinics affiliated with a children's hospital, all in northeastern USA. The STI clinics enrolled individuals aged 18 years and older; the adolescent clinics enrolled individuals as young as 15 . Eligibility criteria included reporting penile-vaginal intercourse in the preceding 3 months; willing to be tested for chlamydia, gonorrhoea and trichomoniasis by providing a urine specimen; speaking English; willing to provide contact information; and providing written informed consent. Institutional review boards at the participating universities approved the study protocol with a waiver of parental consent (only assent was required) for adolescents less than 18 years of age.

Recruitment procedures varied slightly across the five clinics. At the adolescent clinics, the study was listed on a research recruitment flag attached to the appointment paperwork of age-eligible patients. The research assistant used the flag to identify eligible patients. This chart-flagging system at adolescent clinics precluded us from calculating a participation rate for those sites. Across the three remaining clinics, 1424 patients agreed to be screened for eligibility. Of these, 1297 were eligible and invited to participate; 794 enrolled, yielding a participation rate of $61.2 \%$. With the remaining patients from the Boston clinics $(n=135)$, the participant sample size was 929. Data were collected from December 2007 through April 2011.

At baseline, participants completed a gender-specific audio computer-assisted self-interview audio-computer assisted self interviewing (ACASI) assessing their sociodemographic characteristics, sexual history and condom use behaviours, then provided a first-catch urine specimen for STI testing. To optimise their condom use, all participants were offered a brief (30-45 min) gender-specific educational session about using condoms, adapted from a safer sex intervention ${ }^{14}$ designated 'best evidence' by the Centers for Disease Control and Prevention. ${ }^{15}$ A key feature of this interactive counselling programme was learning about the 'fit and feel' of condoms and the pleasure-based aspects of finding the right size and type of condoms, as well as the use of water-based lubricants. Participants were then offered their choice from an extensive selection of condoms and water-based lubricants.

Participants were trained in the use of an electronic diary report using a password-protected personal digital assistant (PDA) programmed with the Configurable Electronic Real-Time Assessment System (CERTAS; Personal Improvement Computer Systems, Inc. Reston, Virginia, USA). Each day, participants were prompted to answer the question of whether they had sex in the past $24 \mathrm{~h}$. Because the research question pertained to urethral/vaginal acquisition of STIs, our assessments concerned penile-vaginal sex. Thus, oral sex was not assessed in this study. Based on formative research, ${ }^{16}$ we defined sex as 'putting the penis in the vagina' and the end of sex as the male orgasm. If participants responded affirmatively, they were asked questions about each instance of sexual intercourse. They were also asked to enter a report about their sexual behaviour directly into the PDA after each time that they had sex to maximise capture of data on every sex event. To avoid duplicative reporting, participants were prompted to indicate on the daily diary if they had already completed a report about a sex event. To minimise loss of data, participants were asked to return the PDA memory card every 30 days, at which time a new one was provided.

At the 3-month and 6-month follow-up interviews, participants completed an ACASI regarding their sexual behaviours in the past 3 months and provided urine specimens for STI testing. Incident STIs detected at the 3-month visit were treated prior to the start of the second 3 months of daily reporting. Participants were offered remuneration in gift cards based on the study activities completed (maximum amount was approximately USD 2.50 per day of observation).

\section{Assessments}

\section{STI testing}

At baseline, 3 months and 6 months, first-catch urine specimens were tested using the Becton Dickinson (Sparks, Maryland, USA) ProbeTec ET C. trachomatis and N. gonorrhoeae Amplified DNA Assay ${ }^{17}$ and the Taq-Man PCR-ELISA for $T$. vaginalis (developed and validated by the research laboratory ${ }^{18}$ ). All urine specimens were processed within $48 \mathrm{~h}$ and shipped to the study laboratory within 7 days. Participants testing positive for any of the three STIs were contacted immediately and asked to schedule an appointment for treatment. Single-dose therapy with CDC-recommended medications was used to maximise treatment effectiveness. ${ }^{19}$ Finally, to capture data on STIs that occurred between follow-up study visits, we conducted a chart review at the end of the observation period for each participant.

\section{Daily measures}

On the daily electronic report, participants who indicated sex in the past $24 \mathrm{~h}$ were asked about each sex event, 'Did you and your partner use a condom for the penis-in-vagina part of this sex event?' If they responded yes, they were asked questions about the condom use, including the occurrence of the following five errors and problems: breakage, slippage during sex, slippage after sex was over, putting the condom on after sex began and taking the condom off before sex ended.

\section{Data analysis}

Data were summarised across each 3-month interval (0-3 months and 3-6 months), with participants contributing either one or two intervals to the analyses. Analyses were restricted to 3-month intervals with at least one sex event report. Consistent condom use was defined as condom use with every sex event. Correct condom use was defined as a 'no' answer to each of the five questions on errors and problems when a condom was used. The association between condom use and incident STI was examined using generalised estimating equation models to account for within-individual correlation of observation intervals. ${ }^{20}{ }^{21}$ Model 1 tested the association between consistent condom use (yes, no) and 3-month incident STI. Model 2 examined the association between condom use and incident STI when correct as well as consistent condom use (yes, no) was required. Both models adjusted for gender, age group (15-19, 20-24, 25+ years) and history of STI (STI reported on baseline ACASI or positive STI test result during the preceding 3-month interval), which were examined individually for significant interactions with the condom use variable. Significance was defined by an $\alpha$ level of 0.05 . Power calculations suggested that $80 \%$ power was available 
Table 1 Demographic information of the sample stratified by city of enrolment $(\mathrm{N}=928)$

\begin{tabular}{|c|c|c|c|}
\hline & $\begin{array}{l}\text { Northeastern city } \\
(\mathrm{n}=269)\end{array}$ & $\begin{array}{l}\text { Midwestern city } \\
(n=248)\end{array}$ & $\begin{array}{l}\text { Southern city } \\
(\mathrm{n}=411)\end{array}$ \\
\hline Mean Age/SD and IQR* & $22.8(7.1)(18-25)$ & $36.3(11.6)(25.5-47)$ & $29.0(9.0)(22-35)$ \\
\hline Female gender & $172(63.9 \%)$ & $126(50.6 \%)$ & $214(52.1 \%)$ \\
\hline Black race & $95(35.3)$ & $198(79.8)$ & $214(52.1)$ \\
\hline $\begin{array}{l}\text { Mean \# sex } \\
\text { partners, 3M† }\end{array}$ & $2.0(1.7)(1-2)$ & $2.9(5.0)(1-3)$ & $3.5(8.6)(1-3)$ \\
\hline $\begin{array}{l}\text { Mean \# sex } \\
\text { partners, LT‡ }\end{array}$ & $14.9(25.9)(4-15)$ & $39.2(44.3)(1-50)$ & $33.7(38.2)(10-40)$ \\
\hline
\end{tabular}

to detect a protective $\mathrm{OR}$ as small as 0.31 for the effect of consistent and correct use and 0.43 for the effect of only consistent use.

\section{RESULTS}

The mean $\pm S D$ age of the participants was $29.2 \pm 10.8$ years. Two-thirds $(\mathrm{n}=617,66.5 \%)$ identified as African-American and more than one-half $(\mathrm{n}=512,55.1 \%)$ were women. The mean \pm SD number of lifetime sex partners was 29.7 \pm 38.2 . Table 1 provides this demographic information stratified by city. Most (65.7\%) of those 18 and older reported earning less than $\$ 1000$ per month in income or social assistance and $54.4 \%$ of those under 18 reported they qualified for a free lunch at school. Just under one-third of the sample (30.7\%) reported ever being diagnosed with a STI. Seven hundred and eight participants $(76.2 \%)$ returned for the initial follow-up assessment visit and 523 returned for a second follow-up assessment.

Three hundred-eighty participants contributed data for two 3-month intervals and 200 contributed data for one 3-month interval, yielding a total of 9603 -month intervals. Of the 380 participants providing data for both intervals, only 99 were classified discrepantly on consistent use between the two intervals. Of these same 380 participants, only 52 were classified discrepantly on consistent and correct use between the two intervals. Of the 960 3-month intervals, 14 (1.5\%) involved indeterminate STI assay results, resulting in 9463 -month intervals for analysis. Table 2 displays further descriptive information for each 3-month interval, stratified by city.

Participants reported a total of 14970 penile-vaginal sex events, of which 9545 (63.8\%) involved use of a condom. Of the sex events involving a condom, 2285 (23.9\%) included one or more condom use errors or problems, leaving 7260 events when condoms were used correctly. Table 3 displays further descriptive information for each 3-month interval, stratified by gender.

Three-month and 6-month assays for incident STIs yielded 116 positive test results (44 for chlamydia, 15 for gonorrhoea and 57 for trichomoniasis). In addition, chart reviews of clinic diagnoses made during study enrolment yielded two cases that were not detected by nucleic acid amplification test at

Table 2 Descriptive information of the sample, shown by 3-month interval of observation, stratified by city of enrolment

\begin{tabular}{|c|c|c|c|c|}
\hline & Northeastern & Midwestern & Southern & Total \\
\hline \multicolumn{5}{|l|}{ Sexual behaviours } \\
\hline Interval $1^{*}$ & $(n=203)$ & $(n=148)$ & $(n=217)$ & $(n=568)$ \\
\hline Mean \# sex partners, $3 \mathrm{M} \dagger$ & $2.4(2.4)(1-3)$ & $3.9(3.4)(2-5)$ & $3.8(3.5)(1-5)$ & $3.3(3.2)(1-4)$ \\
\hline PVI events per person dy $\ddagger 3008 / 21491(0.14)$ & $2293 / 13620(0.17)$ & $3762 / 21735(0.17)$ & $9063 / 56847(0.16)$ & \\
\hline Interval $2 \S$ & $(n=130)$ & $(n=115)$ & $(n=147)$ & $(n=392)$ \\
\hline Mean \# sex partners, 3M† & $2.5(2.6)(1-3)$ & $3.2(3.5)(1-4)$ & $3.0(2.7)(1-4)$ & $2.9(3.0)(1-3)$ \\
\hline PVI events per person dy $\ddagger 1893 / 10885(0.13)$ & $1884 / 10441(0.18)$ & $2260 / 13492(0.17)$ & $6037 / 34788(0.17)$ & \\
\hline \multicolumn{5}{|l|}{ Sexually Transmitted Infections } \\
\hline Baseline Measures & cases/n (\%) & cases/n (\%) & cases/n (\%) & cases/n (\%) \\
\hline Combined prevalent cases & 19/268 (7.1) & $52 / 244(21.3)$ & $38 / 265(14.3)$ & $169 / 920(18.4)$ \\
\hline Prevalent gonorrhoea cases & $0 / 268(0.0)$ & $3 / 244(1.2)$ & $32 / 408(7.8)$ & $35 / 920(3.8)$ \\
\hline Prevalent chlamydia cases & $14 / 268(5.2)$ & $13 / 244(5.3)$ & 49/408 (12.0) & $76 / 920(8.3)$ \\
\hline Prevalent trichomonas cases & $5 / 268(1.9)$ & $36 / 244(14.8)$ & $38 / 408(9.3)$ & $79 / 920(8.6)$ \\
\hline \multicolumn{5}{|l|}{ Interval $1^{*}$} \\
\hline Combined incident cases & $9 / 236(3.8)$ & $17 / 198(8.5)$ & $38 / 265(14.3)$ & $64 / 699(9.2)$ \\
\hline Incident gonorrhoea cases & $1 / 236(0.4)$ & $3 / 198(1.5)$ & $6 / 264(2.2)$ & 10/698 (1.4) \\
\hline Incident chlamydia cases & $5 / 236(2.1)$ & $37 / 198(3.5)$ & $21 / 265(7.9)$ & $33 / 699(4.7)$ \\
\hline Incident trichomonas cases & $3 / 236(1.3)$ & 10/198 (5.1) & $19 / 264(7.2)$ & $32 / 698(4.6)$ \\
\hline Cases w/in CCC users§ & $1 / 39(2.6)$ & $2 / 40(5.0)$ & $1 / 30(3.3)$ & 4/109 (3.7) \\
\hline Cases w/in <CCC users $\mathbb{I}$ & $7 / 164(4.3)$ & $10 / 106(9.4)$ & 25/182 (13.7) & $42 / 452(9.3)$ \\
\hline \multicolumn{5}{|l|}{ Interval $2 \S$} \\
\hline Combined incident cases & $3 / 164(1.8 \%)$ & $14 / 162(8.6 \%)$ & $22 / 186(11.8 \%)$ & $39 / 512(7.6)$ \\
\hline Incident gonorrhoea cases & $0 / 163(0.0)$ & $1 / 162(1.6)$ & $4 / 186(2.2)$ & $5 / 512(1.0)$ \\
\hline Incident chlamydia cases & 3/164 (1.8) & $3 / 162(1.9)$ & $5 / 186(2.7)$ & $11 / 512(2.1)$ \\
\hline Incident trichomonas cases & $0 / 164(0.0)$ & $11 / 162(6.8)$ & $14 / 186(7.5)$ & $25 / 512(4.9)$ \\
\hline Cases w/in CCC users§ & $0 / 28(0.0)$ & $2 / 34(5.9)$ & $0 / 21(0.0)$ & $2 / 83(2.4)$ \\
\hline Cases w/in <CCC users $\pi$ & $2 / 123(1.6)$ & $10 / 96(10.4)$ & $19 / 145(13.1)$ & $31 / 364(8.5)$ \\
\hline
\end{tabular}

$\angle \mathrm{CCC}=$ not reporting CCC.

*Data from first 3 months of the study.

tData from daily diaries collected over a 3-month period.

$\ddagger$ Total number of penile-vaginal sex events divided by total number of observation days

$\S$ Data from months 4 through 6 of the study.

ICCC $=$ Consistent and Correct Condom. 
Table 3 Descriptive information, by gender, regarding sexual behaviours reported by interval of data collection using daily electronic dairies

\begin{tabular}{|c|c|c|}
\hline Behaviour & Males & Females \\
\hline \multicolumn{3}{|l|}{ Interval $1^{*}$} \\
\hline $\begin{array}{l}\text { Valid n, Mean PVI (SD) } \\
\text { and (IQR)† }\end{array}$ & $23115.6(16.1)(4-22)$ & $33716.2(16.1)(5-22$ \\
\hline $\begin{array}{l}\text { Valid } n \text {, Mean PVI partners } \\
\text { (SD) and (IOR) } \ddagger\end{array}$ & $2314.0(3.9)(1-5)$ & $3372.9(2.5)(1-4)$ \\
\hline $\begin{array}{l}\text { Number (\%) of inconsistent } \\
\text { condom use§ }\end{array}$ & $141 / 231(61.0)$ & $228 / 337(67.7)$ \\
\hline Number $(\%)$ of $<$ CCCI & $180 / 231(77.1)$ & 279/337 (82.8) \\
\hline \multicolumn{3}{|c|}{ Condom Errors/Problems (231 males, 337 females) } \\
\hline Breakage: Mean (SD) & $0.4(1.2)$ & $0.5(1.0)$ \\
\hline $\begin{array}{l}\text { Slippage during withdrawal: } \\
\text { Mean (SD) }\end{array}$ & $0.4(1.3)$ & $0.6(1.5)$ \\
\hline $\begin{array}{l}\text { Slippage during PVI: } \\
\text { Mean (SD) }\end{array}$ & $0.3(1.1)$ & $0.6(1.4)$ \\
\hline $\begin{array}{l}\text { Incomplete }{ }^{* *} \text { condom use: } \\
\text { Mean (SD) }\end{array}$ & $1.8(3.3)$ & $1.5(3.0)$ \\
\hline $\begin{array}{l}\text { Not using a new condom: } \\
\text { Mean (SD) }\end{array}$ & $0.5(1.8)$ & $0.5(3.1)$ \\
\hline \multicolumn{3}{|l|}{ Interval $2+\dagger$} \\
\hline $\begin{array}{l}\text { Valid n, Mean PVI (SD) } \\
\text { and (IOR) } \dagger\end{array}$ & $15413.6(14.7)(3-19)$ & $23816.6(17.9)(4-22$ \\
\hline $\begin{array}{l}\text { Valid n, Mean PVI partners } \\
\text { (SD) and (IOR) } \ddagger\end{array}$ & $1543.1(3.2)(1-4)$ & $2382.7(2.8)(1-3)$ \\
\hline $\begin{array}{l}\text { Number (\%) of inconsistent } \\
\text { condom use§ }\end{array}$ & $86 / 154(55.8)$ & $154 / 238(64.7)$ \\
\hline Number (\%) of < CCCI & $124 / 154(80.5)$ & $208 / 238(87.4)$ \\
\hline \multicolumn{3}{|c|}{ Condom Errors/Problems (154 males, 238 females) } \\
\hline Breakage: Mean (SD) & $0.4(1.2)$ & $0.4(1.3)$ \\
\hline $\begin{array}{l}\text { Slippage during withdrawal: } \\
\text { Mean (SD) }\end{array}$ & $0.3(1.1)$ & $0.5(1.6)$ \\
\hline $\begin{array}{l}\text { Slippage during PVI: } \\
\text { Mean (SD) }\end{array}$ & $0.3(0.8)$ & $0.5(1.6)$ \\
\hline $\begin{array}{l}\text { Incomplete }{ }^{* *} \text { condom use: } \\
\text { Mean (SD) }\end{array}$ & $1.3(3.6)$ & $1.4(3.3)$ \\
\hline $\begin{array}{l}\text { Not using a new condom: } \\
\text { Mean (SD) }\end{array}$ & $0.5(2.8)$ & $0.7(6.2)$ \\
\hline
\end{tabular}

${ }^{*}$ Assessed during the first 3 months of the study.

tMean frequency of penile-vaginal sex during 3-month observation interval, with SD and IQR.

$\ddagger$ Mean number of penile-vaginal sex partners during 3-month observation interval, with SD and IQR.

$\S$ Defined as using condoms for less than $100 \%$ of all penile-vaginal sex acts during the interval.

TDefined as not using condoms consistently and correctly for all penile-vaginal sex acts during the interval.

**Defined as putting condoms on after sex had begun or taking condoms off before sex ended.

††Assessed during the second 3 months of the study.

follow-up. Of these 118 cases, 81 (69\%; 31 for chlamydia, 10 for gonorrhoea and 40 for trichomoniasis) had electronic diary data over the corresponding 3-month interval. Accounting for co-infections, there were 74 analysis intervals during which a subject acquired at least one of the three STIs.

To determine whether systematic differences existed between the 74 observation intervals remaining in the analysis and the 29 intervals that were excluded, a comparison was conducted based on the ACASI baseline data. These data allowed us to test for differences in consistent condom use (but not correct use as this had to be assessed in real time) and the three covariates (gender, age and history of STIs). Significant differences for gender $(p=0.27)$, age $(p=0.99)$ and STI history $(p=0.30)$ were not found. However, the 29 intervals excluded were more likely $(p=0.023)$ to include consistent condom use than the 74 intervals in the analysis.

\section{Bivariate associations}

Of the 946 3-month intervals, 603 (63.7\%) involved less-than-consistent use of condoms. STIs occurred in 51 of these 603 intervals, yielding $8.46 \%$ incident infections among those not reporting consistent use during a 3-month interval. Conversely, 343 intervals $(36.3 \%)$ involved consistent use of condoms. STIs occurred in 23 of these intervals, yielding $6.71 \%$ incident infections among those who reported consistent condom use. The difference between these two percentages (absolute difference $=1.75 \%$; percent relative difference $=20.7 \%$ ) was not significant (estimated $\mathrm{OR}=0.73$; 95\% CI (CI) 0.42 to 1.26 ; $\mathrm{p}=0.26$ ).

Of the 946 observation intervals, 777 (82.1\%) involved less-than-consistent and/or incorrect use of condoms. Within these 777 intervals, 68 cases of at least one STI occurred, yielding $8.75 \%$ incident infections among those not reporting consistently and correct use during an observation interval. Conversely, 169 (17.9\%) involved consistent and correct use of condoms. Within these intervals, six cases of at least one STI occurred, yielding $3.35 \%$ incident infections among those reporting consistent and correct use. The difference between these two percentages (absolute difference $=5.20 \%$; percent relative difference $=59.0 \%$ ) was significant (estimated $\mathrm{OR}=0.41$; $95 \%$ CI 0.19 to $0.88 ; p=0.023$ ).

We also investigated the possibility of a linear relationship between the number of unprotected events and the log odds of incident STIs. Bivariate analyses showed that incidence jumped from $3.6 \%(6 / 169)$ with no unprotected or imperfectly protected events, to $9.0 \%(11 / 122)$ with one and to $8.9 \%(20 / 224)$ with between two and four such events, suggesting that, for example, one such event is not $25 \%$ as bad as four such events.

\section{Multivariate analyses}

Table 4 displays the findings from generalised estimating equation analyses modelling the effect of condom use on incident STI over the observation intervals. Adjusted for gender, age group and history of STI, consistent condom use was not significantly protective against acquiring an incident STI (Model 1). Significant interactions were not found between consistent condom use and age group $(p=0.27)$, gender $(p=0.43)$ or STI history $(p=0.22)$.

In contrast to Model 1, participants who used condoms both correctly and consistently were estimated to have 59\% smaller odds of acquiring an STI over 3 months (see Model 2 in table 4) compared to participants who did not use condoms both correctly and consistently, adjusting for gender, age group and history of STI. Significant interactions were not found between consistent and correct condom use and age group $(p=0.60)$, gender $(p=0.59)$ or STI history $(p=0.81)$.

\section{DISCUSSION}

Findings provide a striking contrast between testing condom effectiveness based on consistent use versus a more refined measure accounting for errors and problems. This contrast parallels the concepts of 'typical use effectiveness' and 'perfect use effectiveness' from contraceptive studies. ${ }^{22}$ The discrepancy between typical and perfect use in this study was dramatic, with point estimates being 0.75 and 0.41 , respectively. The implication is that global efforts to promote condom use should be augmented with efforts to promote their correct use. A recent review suggests that condom use errors and problems are a global issue. ${ }^{23}$ Incomplete use of condoms is a problem 
Table 4 Generalised estimation equation (GEE) estimates for the effect of condom use on incident infections with Chlamydia, Gonorrhoea and Trichomoniasis

\begin{tabular}{|c|c|c|c|}
\hline Predictor variable & Point estimate & $95 \% \mathrm{Cl}$ & p Value \\
\hline \multicolumn{4}{|c|}{ Model 1: Effects of consistent condom use only } \\
\hline Consistent condom use & 0.75 & 0.42 to 1.32 & 0.32 \\
\hline Male gender & 0.81 & 0.44 to 1.50 & 0.50 \\
\hline Less than 20 years of age $\dagger$ & 2.00 & 0.99 to 4.03 & 0.05 \\
\hline 20-24 years of age $t$ & 1.11 & 0.57 to 2.17 & 0.76 \\
\hline Past sexually transmitted infections & 2.96 & 1.58 to 5.56 & 0.0001 \\
\hline \multicolumn{4}{|c|}{ Model 2: Effects of consistent and correct condom use } \\
\hline Consistent and correct condom use & 0.41 & 0.19 to 0.90 & 0.026 \\
\hline Male gender & 0.83 & 0.45 to 1.53 & 0.55 \\
\hline Less than 20 years of age $\dagger$ & 2.05 & 1.01 to 4.14 & 0.047 \\
\hline $20-24$ years of age $\dagger$ & 1.12 & 0.57 to 2.17 & 0.75 \\
\hline Past sexually transmitted infections & 2.98 & 1.61 to 5.52 & 0.0005 \\
\hline
\end{tabular}

tThe reference group consists of persons 25 years of age or older.

requiring targeted education. Rectifying issues such as poor fit and feel of condoms and using oil-based lubricants may substantially reduce slippage and breakage. ${ }^{23-29}$

A methodological point of emphasis is that all potential forms of bias to studies of condom effectiveness favour the null hypothesis. ${ }^{6}$ Thus, the perfect use point estimate of 0.41 might have been even lower (but not higher) without bias. Indeed, the six incident cases observed for people using condoms consistently and correctly may be a result of an unprotected sex event, breakage event, etc that was not reported. Collecting self-reported measures is a science that can only be improved, but never perfected, as tendencies to forget, fabricate, exaggerate and under-report are inevitable. $^{10}$

Several analytic points warrant discussion. In cases where partners are uninfected, inconsistent and incorrect condom use are irrelevant because participants cannot acquire infection. In cases with infected partners, pinpointing the transmission date is impractical, thereby creating the question of whether condoms were used consistently prior to, or after, infection. This question is mute when condoms are used consistently throughout the observation period or not used at all. Thus, using the frequency of unprotected sex as a predictor variable, rather than consistent use or correct and consistent use is inherently flawed. Finally, the absence of sexual event data for 29 intervals in which STIs were acquired was an unfortunate reality. That these intervals included disproportionate numbers of consistent condom users suggests further bias toward the null.

The protective OR of 0.41 is remarkably similar to the 0.42 reported by Warner and colleagues against chlamydia and gonorrhoea. ${ }^{9}$ Only our estimate was obtained with accounting for the correct use of condoms. Further, Warner and colleagues did not assess $T$. vaginalis infection. Thus, our findings support and extend their findings.

An important implication of our findings pertains to the lack of significance for consistent use (unadjusted for correct use). This suggests that efficacy trials of safer sex programmes should be evaluated using the metric of consistent use and correct condom use. Failure to account condom use problems/ errors could result in an effective intervention being incorrectly deemed ineffective. Also, based on imperfect protection offered by typical condom use, findings support the case of using biomarker outcomes (ie, STI acquisition) in efficacy trials of HIV prevention programmes. Failure to do so may result in an ineffective intervention being incorrectly deemed effective.

\section{Limitations}

The study was not adequately powered to analyse condom effecti veness separately for chlamydia, gonorrhoea and trichomoniasis. Also, any prospective study of condom effectiveness against nonviral STIs precludes the possibility of knowing the infection status of sex partners. Thus, the estimate of 0.41 must be considered conservative. Further, misclassification bias from inaccurate reporting may have also led to conservative point estimates. ${ }^{7} 9$ Finally, it is well worth noting that sample bias is not a primary issue in this type of study given that the basic research question is biological/physical rather than behavioural.

\section{Conclusion}

In this largest study to-date designed specifically to test the effectiveness of condoms against non-viral STIs, we observed that consistent and correct use of condoms reduces the estimated odds of an infection by almost $60 \%$, with no significant differences in effectiveness by age group, gender or STI history. Magnified over an entire population, this level of risk reduction for sexually active people is substantial. In this study, almost $18 \%$ of analysis intervals entailed using condoms consistently and correctly-intervention efforts to raise that value to $59 \%$ (corresponding to conversions on half of the analysis intervals not entailing consistent and correct use) would be anticipated to avert approximately 21 infections per 1000 people over a 3-month interval.

\section{Key messages}

- Whether condoms confer significant protection against non-viral STIs is a function of their correct use. Incomplete use is especially common and therefore problematic.

- The consistent and correct use of condoms provides excellent protection against non-viral STI acquisition.

- The point estimate of 0.41 is conservative in that multiple forms of bias toward the null are common and unavoidable in studies of condom effectiveness.

- Trials of safer sex programmes should always be evaluated using the metric of consistent and correct condom use.

Acknowledgements We wish to thank our advisory board members for the guidance: Drs Ward Cates, Jonathan Zenilman and Ralph DiClemente. Also, we are grateful to our research assistants-Margaret Reed, Rachel Vickers, Lisa Sunner, Christopher Lops, Amanda Wallace, Ashley Kendall and Devika Bhushan.

Contributors RA Crosby acquired funding for the study, served as PI, provided oversight of all data collection procedures, drafted the initial manuscript and revised the final manuscript. RA Charnigo served as the study statistician, provided oversight of data collection and cleaning, conducted all analyses and participated in manuscript preparation. CW served as the Project Director and thus provided oversight for data collection and assured adherence to study protocols in all locations. AMC supervised the laboratory responsible for nucleic acid amplification testing of all urine specimens. LAS assisted RA Crosby in obtaining funding, provided scientific guidance, designed protocols for use of PDAs and CERTAS and participated in manuscript preparation.

Funding Supported by a grant from NIAID to Dr Crosby (RA1068119A) and this work was supported in part by the Center for AIDS Research (P30 AI050409).

Competing interests None.

Ethics approval University of Kentucky Institutional Review Board.

Provenance and peer review Not commissioned; externally peer reviewed 


\section{REFERENCES}

1. United States Department of Health and Human Services. Workshop Summary: Scientific evidence on condom effectiveness for sexually transmitted disease (STI) prevention. http://www.niaid.nih.gov/dmid/STIs/condomreport.pdf (accessed 5 Jul 2011).

2. Wald A, Langenberg AGM, Link K, et al. Effect of condoms on reducing the transmission of herpes simplex virus type 2 from men to women. JAMA 2001;285:3100-6.

3. Warner L, Macaluso M, Austin HD, et al. Application of the case-crossover design to reduce unmeasured confounding in studies of condom effectiveness. Am J Epidemiol 2005;161:765-73.

4. Koss CA, Dunne EF, Warner L. A systematic review of epidemiologic studies assessing condom use and risk of syphilis. Sex Transm Dis 2009;7:401-5.

5. Winer RL, Hughes JP, Feng 0 , et al. Condom use and the risk of genital human papillomavirus infection in young women. NEJM 2006;354:2645-54.

6. Crosby RA, Bounse S. Condom effectiveness: where are we now? Sex Health 2011;9:10-7.

7. Crosby RA, DiClemente RJ, Holtgrave DR, et al. Design, measurement, and analytic considerations for testing hypotheses relative to condom effectiveness against non-viral STIs. Sex Transm Infect 2002;78:228-31.

8. Crosby RA, Salazar LF, DiClemente RJ, et al. Accounting for failures may improve precision: evidence supporting improved validity of self-reported condom use. Sex Transm Dis 2005;32:513-5.

9. Warner L, Newman DR, Fishbein $\mathbf{M}$, et al. Problems with condom use among patients attending sexually transmitted disease clinics: prevalence, predicators, and relation to incident gonorrhea and chlamydia. Am J Epidemiol 2008;167:341-9

10. Zimmerman RS, Atwood KA, Cupp PK. Improving validity of self-reports for sensitive behaviors. In: DiClemente RJ, Crosby RA, Salazar LF, Eds. Research methods for health promotion. San Francisco, CA: Jossey-Bass Wiley, 2006 : 229-59.

11. Fairley CK, Sze JK, Vodstrcil LA, et al. Computer-assisted self interviewing in sexual health clinics. Sex Transm Dis 2010;37:665-8.

12. Peterman TA, Lin LS, Newman DR, et al. Does measured behavior reflect STD risk? an analysis of data from a randomized controlled behavioral intervention study. Sex Transm Dis 2000;27:446-51.

13. Warner L, Newman DR, Austin HD, et al. Condom effectiveness for reducing transmission of gonorrhea and Chlamydia: the importance of assessing partner infection status. Am J Epi 2004;159:242-51.
14. Crosby RA, DiClemente RJ, Charnigo R, et al. A brief, clinic-based, safer sex intervention for African American men at-risk of HIV acquisition: a randomized controlled trial. Am J Public Health 2009;99:S96-S103.

15. Centers for Disease Control and Prevention. Compendium of Evidence-Based HIV Prevention Interventions. 2009; http://www evidence-interventions.htmצhash; completelist. (accessed 21 Oct 2009).

16. Mehta CM, Sunner L, Head S, et al. 'Sex isn't something you do with someone you don't care about': adolescent females definitions of sex. J Pediatr Adolesc Gynecol 2011;24:266-71.

17. Van der Pol BD, Ferrero L, Buck-Barrington E, et al. Multicenter evaluation of the BDProbeTec ET system for detection of Chlamydia trachomatis and Neisseria gonorrhoeae in urine specimens, female endocervical swabs, and male urethral swabs. J Clin Micro 2001;39:1008-16.

18. Caliendo AM, Jordan JA, Green AM, et al. Real-time PCR improves detection of Trichomonas vaginalis infection compared with culture using self-collected vaginal swabs. Infect Dis Obstest Gyn 2005;13:145-50.

19. Workowski KA, Berman S. Sexually transmitted diseases treatment guidelines, 2010. MMWR 2010;59 (RR-12) 1-110.

20. Liang K-Y, Scott Z. Longitudinal data analysis using generalized linear models. Biometrika 1986;73:13-22.

21. Hardin J, Hilbe J. Generalized estimating equations. London: Chapman and Hall/CRC, 2003

22. Hatcher RA, Trussel J, Nelson AL, et al. Eds. Contraceptive technology. 19th revised edn. New York, NY: Ardent Media, 2007.

23. Sanders SA, Yarber WL, Kaufman EL, et al. Condom use errors and problems: a global view. Sexual Health 2011;9:81-5.

24. Crosby RA, Yarber WL, Sanders SA, et al. Men with broken condoms: who and why? Sex Transm Infect 2007;83:71-5.

25. Crosby RA, Graham CA, Yarber WL, et al. If the condom fits, wear it: a qualitative study of young African American men. Sex Transm Infect 2004;80:306-9.

26. Crosby RA, Yarber WL, Sanders SA, et al. Condom discomfort and associated problems with their use among university students. Am J College Health 2005;54:143-8.

27. Reece M, Herbenick D, Sanders SA, et al. Breakage, slippage and acceptability outcomes of a condom fitted to penile dimensions. Sex Transm Infect 2008;84:143-9.

28. Crosby RA, DiClemente RJ, Yarber WL, et al. An event-specific analysis of condom breakage among African American men at risk of HIV acquisition. Sex Transm Dis 2008;35:174-8.

29. Yarber WL, Graham CA, Sanders SA, et al. Correlates of condom breakage and slippage among university undergraduates. Int J STDs and AIDS 2004;15:467-72. 